\title{
Isolated Fallopian Tube Torsion in a Premenarchal Female
}

Melissa Duignan*, James Young and Ashlee Smith

Geisinger Medical Center, USA

\begin{abstract}
Background: Torsion of an ovary or fallopian tube is a surgical emergency. A delay in diagnosis and treatment may lead to sepsis, compromise of fertility, or even death. Isolated fallopian tube torsion is a rare occurrence in the adolescent population. They are difficult to diagnose, as they typically do not present with the prodromal symptoms that are typically seen with an ovarian torsion.
\end{abstract}

Case description: An $11 \mathrm{~cm}$ pelvic mass was diagnosed in an eleven-year-old female who presented with nonspecific lower abdominal pain. The mass was found intraoperatively to be an isolated fallopian tube torsion, which spared the ovary. A salpingectomy was performed, leaving the healthy ovary in place.

Conclusion: Although a rare occurrence, isolated fallopian tube torsion must be considered in the differential diagnosis for non-specific abdominal pain in the adolescent population. Early surgical intervention is important for salvaging tissue as well as the patient's fertility.

Keywords: Fallopian tube; Gynecology; Fertility

\section{Introduction}

Torsion of an ovary or fallopian tube is a surgical emergency. If not promptly diagnosed and treated, it may lead to sepsis, compromise of fertility, or even death. Isolated fallopian tube torsion is a rare occurrence in the adolescent population. They are difficult to diagnose as they typically do not present with the prodromal symptoms that are seen with an ovarian torsion [1]. Therefore, diagnosis is usually delayed and the patients' health and possibly, fertility may be compromised. Herein, we present a case of isolated fallopian tube torsion in an eleven year old female presenting with non-specific abdominal pain.

\section{Case Description}

An eleven year old premenarchal female presented with complaints of right lower abdominal pain. A CAT scan of her abdomen and pelvis demonstrated an $11.4 \mathrm{~cm}$ pelvic mass as well as a slightly enlarged left ovary. Initially, the patient was discharged home with pain medication and no further work up.

The pain continued to worsen and she presented to the Emergency Department. Differential diagnosis at this point included a large para tubal cyst, ovarian torsion and ovarian malignancy. Upon her arrival, the patient received analgesia and an MRI was performed to further delineate the nature of the pelvic mass. The MRI findings were consistent with a torsed infundibulopelvic

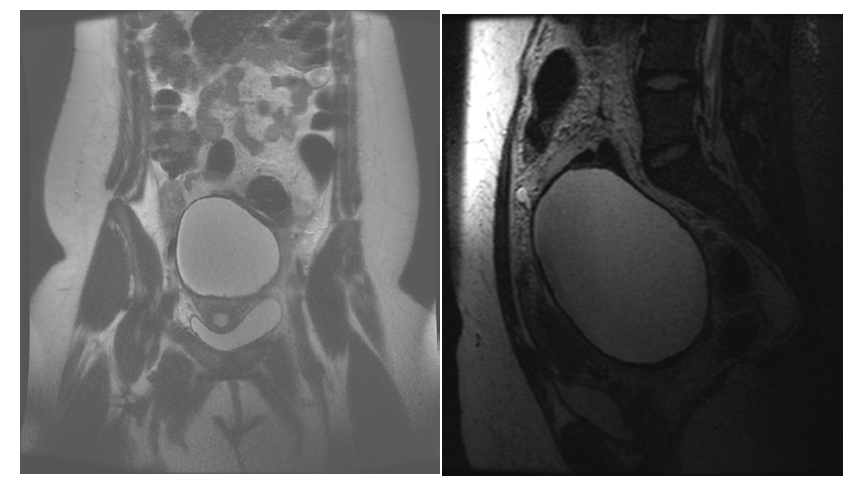

Figure 1: MRI imaging of paratubal cyst in 11 year old female (Left: Coronal view; Right: Sagittal). pedicle and a left para-ovarian simple cyst measuring $9.7 \times 9.4 \mathrm{~cm}$ (Figure 1). There was concern for ovarian torsion, with possible infarction. No further imaging studies were indicated at this time.

The patient was taken to the operating room shortly thereafter for a diagnostic laparoscopy and possible left salpingo-oophorectomy. Upon laparoscopic entry, a necrotic mass was obstructing the majority of the abdominal cavity (Figure 2). The ovaries were noted to be grossly normal bilaterally and the point of torsion was identified at the base of the left fallopian tube. Of note, the bladder, uterus, and appendix were grossly normal. A left salpingectomy was performed, leaving the healthy ovary in place.

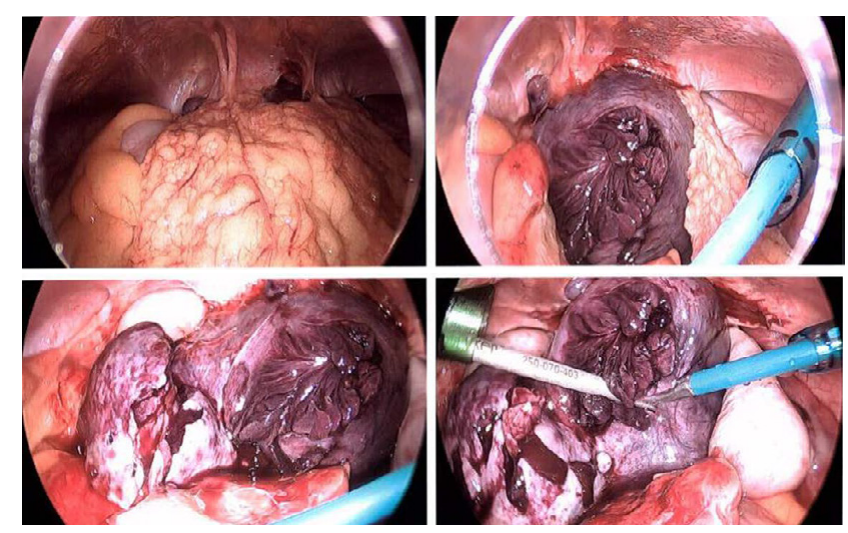

Figure 2: Laparoscopic images - Large obstructing mass with noted adhesions (top left and right).

Isolated fallopian tube torsion with bilaterally healthy ovaries (bottom left and right).

*Corresponding author: Melissa Duignan, Geisinger Medical Center, USA, Tel: 516-319-1386; E-mail: maduignan@geisinger.edu

Received November 11, 2014; Accepted December 15, 2014; Published December 15, 2014

Citation: Duignan M, Young J, Smith A (2014) Isolated Fallopian Tube Torsion in a Premenarchal Female. J Clin Case Rep 4: 461. doi:10.4172/2165-7920.1000461

Copyright: (c) 2014 Duignan M, et al. This is an open-access article distributed under the terms of the Creative Commons Attribution License, which permits unrestricted use, distribution, and reproduction in any medium, provided the original author and source are credited. 
Citation: Duignan M, Young J, Smith A (2014) Isolated Fallopian Tube Torsion in a Premenarchal Female. J Clin Case Rep 4: 461. doi:10.4172/2165-7920.1000461

The patient's post-operative course was uncomplicated and she was discharged to home in stable condition on POD\#1. The final pathology revealed fibro connective and adipose tissue with extensive hemorrhagic necrosis consistent with fallopian tube torsion.

\section{Discussion}

Although a rare occurrence, torsion of the fallopian tube must be considered in the differential diagnosis for abdominal pain in the adolescent population. The diagnosis is frequently delayed secondary to its rarity and the expansive work-up generalized abdominal pain entails. The reported incidence of isolated fallopian tube torsion is approximately 1 in 1.5 million [2]. Early surgical intervention is important for salvaging tissue as well as the patient's fertility.

The pathophysiology related to torsion of the fallopian tube or ovary involves the structural integrity of human blood vessels. Arteries, which are more rigid and have muscular walls, are not easily compressed. They are able to maintain their patency despite twisting on an axis. Veins, however, are thin-walled and once twisted on an axis, collapse and can no longer provide an outflow tract for blood [3]. Upon occurrence of ovarian or fallopian tube torsion, the ovarian or tubal branches of the uterine vein get compressed and therefore the blood continues to back up into said organ. Therefore, blood can flow in but cannot flow out. This leads to further torsion, progression to necrosis, and worsening prognosis.

The etiology of isolated fallopian tube torsion is not clearly understood. Possible inciting factors that are intrinsic to the fallopian tube include previous tubal ligation, hydrosalpinx, congenital anomalies, and tubal neoplasms while extrinsic factors include a history of pelvic inflammatory disease, trauma and pregnancy [4].

Fallopian tube torsion remains difficult to diagnosis. The clinical presentation may be vague; in fact, most patients are completely asymptomatic in the early stages. If symptoms are present, the most common presenting complaint is sharp lower abdominal pain that radiates to the side of the torsion. Other presenting signs and symptoms may be nausea, vomiting, diarrhea, fever and leukocytosis [5]. Imaging remains a challenge for radiologists because it is difficult to discern the exact axis upon which the mass twisted. If ultrasound is performed, the sonographic findings should be interpreted with the patient's clinical history in mind. Possible imaging findings include a dilated fallopian tube, a normal-appearing ipsilateral ovary and presence or absence of Doppler flow [6].

It is possible, if caught early, to reverse the torsion and drain the associated cyst [7]. Therefore, it is important to always maintain a high index of suspicion of isolated fallopian tube torsion when creating a differential diagnosis for non-specific abdominal pain. Without expediting treatment, the fallopian tube may undergo irreversible damage and compromise the patient's future fertility.

\section{References}

1. Rajaram S, Bhaskaran S, Mehta S (2013) Isolated fallopian tube torsion in adolescents. Case Rep Obstet Gynecol 2013: 341507.

2. Gross M, Blumstein SL, Chow LC (2005) Isolated fallopian tube torsion: a rare twist on a common theme. AJR Am J Roentgenol 185: 1590-1592.

3. Kardakis S, Barranca A, Vitelli A, Amore I, Trento F, et al. (2013) Isolated fallopian tube torsion. Case Rep Obstet Gynecol 2013: 479698.

4. Phillips K, Fino ME, Kump L, Berkeley A (2009) Chronic isolated fallopian tube torsion. Fertil Steril 92: 394

5. Ericki, Hosgor Isolated Salpingeal Torsion in Children: A case series.

6. Comerci G, Colombo FM, Stefanetti M, Grazia G (2008) Isolated fallopian tube torsion: a rare but important event for women of reproductive age. Fertil Steril 90: 1198.

7. Rachel K Casey, Lauren F Damle, Veronica Gomez Lobo (2013) Isolated Fallopian Tube Torsion in Pediatric and Adolescent Females: A Retrospective Review of 15 cases at a Single Institution. Journal of Pediatric and Adolescent Gynecology 26: 189-192 